\title{
RADIO SOURCE VARIABILITY AS A TOOL FOR VERY HIGH RESOLUTION
}

\author{
N. SHAPIROVSKAYA (1), O. B. SLEE (2), P. HUGHES (3), \\ G. TSAREVSKY $(1,2)$ \\ (1)Astro Space Centre, Moscow, Russia \\ (2)Australia Telescope National Facility, CSIRO, Sydney \\ (3)Astronomy Department, University of Michigan, Ann Arbor, USA
}

\begin{abstract}
If flux density variability is intrinsic to extragalactic sources, then the shortest time scale of the variability yields an extremely high angular resolution. There is, however, good evidence that some of the variability is due to refractive scintillation in our galaxy's interstellar plasma turbulence. The effect, initially discovered at low radio frequencies, is here shown to extend into the $\mathrm{GHz}$ band, which, up to now, has been assumed to display only the intrinsic variability. We conclude that further study in both the intermediate and high frequency bands needed to separate the intrinsic and extrinsic components of variability.
\end{abstract}

\section{Introduction}

Flux density variation in extragalactic radio sources may be connected with either the output of the central engine or with propagation effects in the three intervening media - the latter are gas in the source itself, the intergalactic medium, and the interstellar medium.

Theoretical considerations suggest that whether the variability mechanism be intrinsic or extrinsic, the angular diameter of the source must be extremely small. For example, light-travel-time arguments suggest that for intrinsic variability with time scale of about 1 year the angular diameter of a source with a redshift $z=2$ will be not more than $6 * 10^{-5}$ arc sec. In the case of refraction and/or diffraction in the interstellar turbulent plasma medium, the source diameter must be less that the diameters of the diffracting or refracting ionized clouds of gas. Such a source must scintillate similar to scintillation of pulsar (see Shapirovskaya, 1991). 
There is reasonable strong evidence that a considerable part of source variability, especially at low radio frequencies $(<1000 \mathrm{MHz})$, is due to diffraction or refraction in the interstellar turbulence. Studies of pulsar scintillation (Arlurkar et al., 1986) and radio sources broadening (Rao and Slee, 1988) have shown that turbulence in the ionized interstellar medium increases towards the galactic plane and towards the galactic centre. Therefore it is reasonable to expect a dependence of source variability on galactic co-ordinates if propagation effects in the interstellar medium take place. Such variability usually expressed in the term of modulation index defined as the standard deviation related to the averaged flux over all measurements of that source.

Shapirovskaya et al (1992) have already shown that the median modulation indices of a large sample of extra galactic sources observed at irregular intervals from 1972 to 1984 with the Culgoora array at 80 $\mathrm{MHz}$ and $160 \mathrm{MHz}$ tend to be systematically higher towards the galactic centre that in the anti-centre region. Dividing this sample into three sub samples corresponding to the centre, anti-centre and poles regions has lead to a similar tendency, see Table 1.

\section{Evidence for variability asymmetry at the $5 \mathrm{GHz}$ band}

We report here that a galactic-coordinate dependence may also be present at much higher frequencies. We have calculated variability indices for a considerable sample of sources (about 80 from 97 observed for which more than 20 observations exist) that were monitored systematically from 1977 by a group at the University of Michigan Radio Observatory (Aller et al., 1985). Table 1 shows these results from their $4.8 \mathrm{GHz}$ measurements, together with above mentioned low frequency results. Both sets of indices are based on variations taking place with time scale 1 year or more.

Table 1 suggests that despite the low levels of statistical significance of the differences in median modulation index between the differing galactic directions (about $P=0.2$ for each individual frequency), there is a definite tendency for this index to increase from the poles to the anti-centre and then to the centre. 
Table 1 Modulation Indices for Sources in the Centre, Anti-Centre and Poles Galactic regions for the Culgoora (80 $\mathrm{MHz}$ and $160 \mathrm{MHz}$ ) and Michigan Radio Observatory (4,800 MHz) monitoring programmes

\begin{tabular}{|c|c|c|c|c|c|c|}
\hline \multirow{2}{*}{$\begin{array}{l}\text { Freq. } \\
\mathrm{MHz} \\
\end{array}$} & \multicolumn{2}{|c|}{$\begin{array}{c}\text { Galaxy Centre } \\
1<=45^{\circ} \\
\mid \text { b } \mid<=45^{\circ}\end{array}$} & \multicolumn{2}{|c|}{$\begin{array}{c}\text { Anti-Centre } \\
135^{\circ}<1<225^{\circ} \\
\mid \text { b } \mid<=45^{\circ}\end{array}$} & \multicolumn{2}{|c|}{$\begin{array}{c}\text { Galaxy Poles } \\
0^{\circ}<1<360^{\circ} \\
\mid \text { b } \mid<45^{\circ}\end{array}$} \\
\hline & $\mathrm{m}$ & $\mathbf{N}$ & $\mathrm{m}$ & $\mathbf{N}$ & $\mathrm{m}$ & $\mathbf{N}$ \\
\hline 80 & 0.32 & 9 & 0.28 & 7 & 0.26 & 15 \\
\hline 60 & 0.33 & 16 & 0.31 & 12 & 0.26 & 20 \\
\hline 4,800 & 0.13 & 7 & 0.10 & 27 & 0.08 & 29 \\
\hline
\end{tabular}

Note: $\mathrm{m}$ - median long-scale modulation index;

$\mathrm{N}$ - number of sources in the sample.

\section{Conclusion}

We believe that it is possible to interpret the behaviour shown in Table 1 in terms of the existing refractive scintillation theory (Rickett, 1984), although the latter may need to be modified to extend it up to several $\mathrm{GHz}$ frequencies. Further study of the updated Michigan observations at 8 and $14.5 \mathrm{GHz}$, together with polarized flux density measurements may be of great interest.

\section{REFERENCES}

Aller H.D., Aller M.F., Latimer G., Hodge P.E.: 1985, Astroph. J. Suppl., 59, 513

Arlurkar S.K., Slee O.B., Bobra A.D.: 1986, Aust. J. Phys, 39, 433

Rao A.P., Slee O.B.: 1988, Mon. Not. R. Astron. Soc., 235, 1313

Rickett B.J.: 1986, Astrophys. J., 307, 442

Shapirovskaya N.: 1991, Sov. Astron. Lett., 17, 380

Shapirovskaya N., Slee O.B., Tsarevsky G.: 1992, Proceedings of the International SARA Conference, Manchester (in press) 